\title{
Ultrasound Probe Tracking for Real-Time Ultrasound/MRI Overlay and Visualization of Brain Shift
}

\author{
David G. Gobbi ${ }^{1}$, Roch M. Comeau ${ }^{2}$, and Terry M. Peters ${ }^{1,2}$ \\ 1 Imaging Research Laboratories, John P. Robarts Research Institute \\ University of Western Ontario, London ON N6A 5K8, Canada \\ dgobbi@irus.rri.on.ca \\ tpeters@irus.rri.on.ca \\ 2 McConnell Brain Imaging Centre \\ Montreal Neurological Institute, Montréal QC H3A 2B4, Canada \\ roch@nil.mni.mcgill.ca \\ http://www.bic.mni.mcgill.ca/research/groups/igns/
}

\begin{abstract}
Stereotactic techniques are prevalent in neurosurgery. A fundamental assumption of stereotaxis is that the brain is a rigid body. It has been demonstrated, however, that following a craniotomy the brain tissue will shift by $10 \mathrm{~mm}$ on average. We are investigating intra-operative ultrasound, using an optical tracking system to record the position and orientation of the ultrasound probe, as a method of measuring and correcting for brain shift. We have determined that the accuracy to which ultrasound image coordinates can be tracked (including the errors involved in calibration) is better than $0.5 \mathrm{~mm}$ within the ultrasound image plane, and better than $2 \mathrm{~mm}$ perpendicular to the plane. We apply two visualization methods to compare the ultrasound and the pre-operative MRI: the first is real-time overlay of the ultrasound with the co-planar MR slice, and the second is the real-time texture mapping of the ultrasound video into a 3D view with the MRI. Our technique is demonstrated on a poly vinyl alcohol cryogel phantom.
\end{abstract}

\section{Introduction}

\subsection{Stereotaxis and Brain Tissue Shift}

Pre-operative MR or CT images are used to identify the surgical target and to plan procedures in stereotactic neurosurgery. The images are registered to a surgical coordinate system through a set of fiducial markers visible in the images. The most commonly used fiducials are $\mathrm{Z}$ bars attached to the stereotactic frame: a single MR or CT slice through three or more Z bars is sufficient to calculate the coordinate transformation from image coordinates to stereotactic frame coordinates.

Recently, frameless stereotactic systems have been developed which use fiducial markers affixed directly to the skin or the skull. These markers are registered 
to a 3D tracking system after the patient's head has been immobilized for surgery. The tracking system can then report tool positions in image coordinates.

There is an implicit assumption for both of the above stereotactic methods that the target does not move between the time the pre-operative images are obtained and the time when surgery is performed. This assumption has recently been examined [1][2]. It has been found that for craniotomies, brain tissue will shift by an average of ten millimetres during the operation.

A very general method for dealing with the problem of brain shift is to obtain images during surgery. Imaging modalities that can be used during neurosurgery include interventional MRI and ultrasound.

\section{$1.2 \quad$ Intra-Operative Imaging}

For an intra-operative imaging technique to be acceptable, the benefit it provides must outweigh the disruption it causes to routine operating room activity.

Interventional MR units restrict the access the surgeon has to the patient. Much of the standard operating-room equipment is incompatible with strong magnetic fields, including surgical instruments, anaesthesia units and life-support equipment. In addition to the cost of the interventional MR unit itself, the cost of rendering ancilliary equipment MR compatible is high.

Intra-operative ultrasound can be used as an adjunct to existing neurosurgical procedures because it is safe and minimally disruptive. Some advantages of ultrasound are: 1) a conventional ultrasound machine can be used in the operating room, 2) there are no compatibility problems between ultrasound imaging and standard operating room equipment, 3) the ultrasound machine is portable.

The aim of our group and others that work with stereotactic intraoperative ultrasound [3][4][5] is to use ultrasound images to measure brain shift. This is achieved by tracking the position and orientation of the ultrasound probe, such that the ultrasound image is always registered to the stereotactic coordinate system and to the pre-operative images. We sample the probe position/orientation at $20-60 \mathrm{~s}^{-1}$, which makes a real-time MR/ultrasound overlay possible. A still from an overlay done during a surgical procedure at the Montreal Neurological Institute is shown in Figure 1. The ventricles and the falx were used as landmarks to detect brain shift.

Our ultimate goal is to use homologous landmarks in 3D ultrasound and preoperative 3D data sets to correct the pre-operative images for brain shift via an elastic deformation. We have demonstrated, using a deformable phantom, the $2 \mathrm{D}$ deformation of MR images to match ultrasound images [6].

\subsection{Tracking Systems and Registration}

In order for a tracking system to be useful, two separate calibrations must be performed. The first is required because the coordinate system associated with the surgical tool (e.g. ultrasound image coordinates if the 'tool' is an ultrasound probe) is in general not identical to the coordinate system of the 'tool holder' 

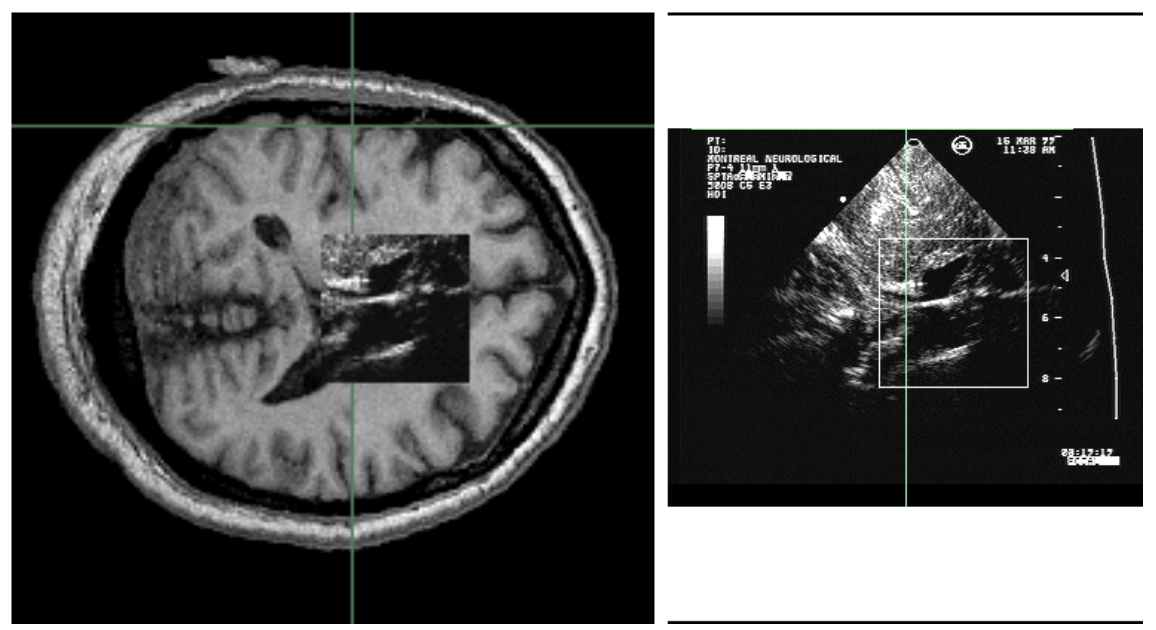

Fig. 1. Left: A still from a MR/ultrasound video overlay which was part of an amygdalohippocampotomy procedure. Right: The ultrasound video still from the overlay, with the overlay ROI outlined.

(the portion of the OTS on which the tracked infared LEDs are mounted). We refer to the transformation between 'tool' and 'tool holder' coordinates as the tool calibration matrix, and denote it ${ }_{\mathrm{u}}^{\mathrm{h}} T$. The procedure we use to find ${ }_{\mathrm{u}}^{\mathrm{h}} T$ for the ultrasound probe is described below.

The second calibration transformation converts tracking-system coordinates (relative to the cameras of the OTS) to a more useful coordinate space (e.g. the pre-operative image space if we are doing an MR/ultrasound overlay). We refer to this transformation as the world calibration matrix, and denote it as ${ }_{\mathrm{t}}^{\mathrm{p}} T$.

These two transformation matrices are combined with the transformation ${ }_{\mathrm{h}}^{\mathrm{t}} T$ returned by the OTS (the transformation between the infrared LEDs and the OTS cameras) to provide the transformation ${ }_{\mathrm{u}}^{\mathrm{p}} T$ between ultrasound image space and pre-operative image space:

$$
{ }_{\mathrm{u}}^{\mathrm{p}} T={ }_{\mathrm{t}}^{\mathrm{p}} T_{\mathrm{h}}^{\mathrm{t}} T{ }_{\mathrm{u}}^{\mathrm{h}} T
$$

\section{Materials and Methods}

The primary components of our system are an Aloka SSD-2000 ultrasound scanner with a $5 \mathrm{MHz}$ neuro ultrasound probe (Aloka Co., Ltd, Tokyo 181-8522, Japan), a POLARIS optical tracking system (Northern Digital Inc., Waterloo ON N2V 1C5, Canada, www.ndigital.com), and a $450 \mathrm{MHz}$ Pentium II workstation with video input/output capabilities.

Our image-guided neurosurgery platform, ASP, has been developed using the Visualization Toolkit [8] (VTK). We have added our own classes to VTK to support the POLARIS, video input, and trilinear interpolation of oblique 
slices from rectilinear image volumes. We chose VTK because it provides a very high-level interface for 3D rendering (making it exceptional for prototyping software), because the source code is available and the addition of custom features is straightforward, and because it is platform-independent across all modern versions of Windows and UNIX.

Our visualization methods were tested on a poly vinyl alchohol cryogel [9] (PVA-C) phantom which we built for that purpose.

\subsection{Ultrasound Probe Calibration}

It is necessary to determine the calibration transformation which converts ultrasound image coordinates to the tracked infrared LED ('tool holder') coordinates. Our calibration relies on a small phantom, consisting of strings supported by a lucite frame, which is modelled after the $\mathrm{Z}$ bars used for frame stereotaxy. The cross section through each $\mathrm{Z}$ bar is visible as three bright spots in the image. We manually place a $2.5 \mathrm{~mm}$ diameter region-of-interest around each spot, within which the centroid is calculated, to measure the coordinates of the spots.

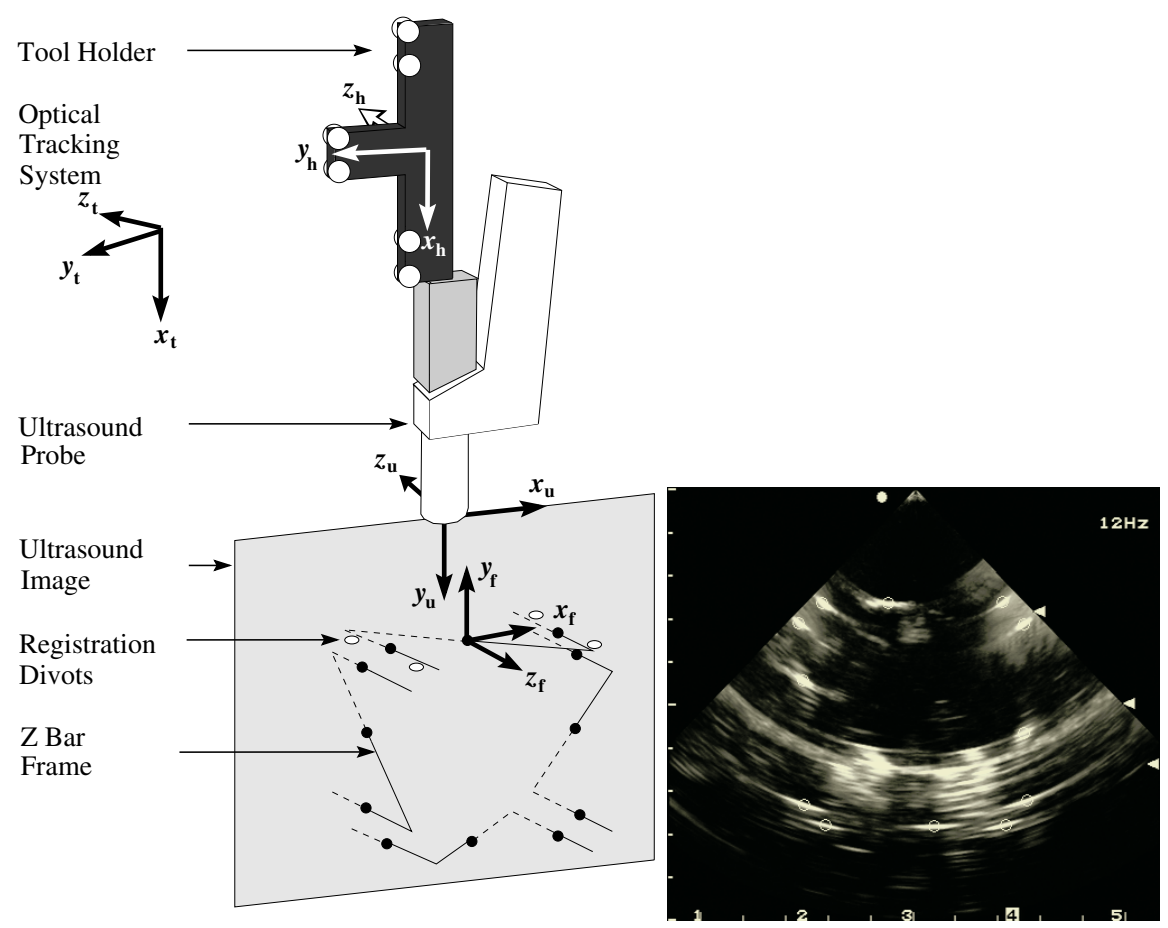

Fig. 2. Ultrasound probe calibration using a Z-bar phantom. One of the annotated images used for a calibration is shown on the right. 
Figure 2 displays the coordinate systems involved in the calibration. The calibration matrix to be found is ${ }_{\mathrm{u}}^{\mathrm{h}} T$, the transformation from ultrasound image coordinates to the tool holder coordinates. The following method is applied:

1. The transformation ${ }_{\mathrm{f}}^{\mathrm{t}} T$ from frame coordinates to tracking system coodinates is calculated, using four divots at known locations on the frame.

2. For each of the point triplets ( $\mathrm{Z}$ bar cross-sections) visible in the image, the position $\mathbf{p}_{\mathrm{f}}$ of the central point in frame coordinates is calculated.

3. Each point is converted to probe-holder coordinates using $\mathbf{p}_{\mathrm{h}}={ }_{\mathrm{t}}^{\mathrm{h}} T \mathrm{f}_{\mathrm{f}}^{\mathrm{t}} T \mathbf{p}_{\mathrm{f}}$.

4. For each point $\mathbf{p}_{\mathrm{h}}$ in probe-holder coordinates, there is a homologous point $\mathbf{p}_{\mathrm{u}}$ in ultrasound image coordinates which is the central point of the corresponding Z-bar cross-section triplet. Least-squares minimization is used to calculate the transformation ${ }_{\mathrm{u}}^{\mathrm{h}} \mathrm{T}$ between ultrasound image coodinates and probe holder coordinates.

We have encapsulated the above procedure within a small application which allows the user to grab ultrasound images (the OTS transformation ${ }_{\mathrm{t}}^{\mathrm{h}} T$ is automatically recorded at the same time), place annotation marks, and apply the above calibration procedure.

\section{$2.2 \quad$ Viewing Options}

Our software currently supports $2 \mathrm{D}$ and $3 \mathrm{D}$ real-time viewing options. In $2 \mathrm{D}$ mode, the tracking of the ultrasound probe is used to determine the intersection of the ultrasound image with the pre-operative MR data set. The MR slice which lies at the intersection is interpolated from the MR data set, and is overlaid on top of the live ultrasound video. The overlay is streamed to a video encoder and can be recorded on a VCR. The typical update rate is 4 frames per second. The result is as shown in Figure 1.

For the $3 \mathrm{D}$ viewing mode (see Figure 3 ), the ultrasound video is streamed into ASP, our 3D surgical navigation software, and an alpha (transparency) mask is applied to it. The ultrasound video is then texture-mapped onto a plane within the $3 \mathrm{D}$ scene, which is re-rendered with an updated video frame and ultrasound probe position at approximately ten frames per second.

\section{Results and Discussion}

\subsection{POLARIS Accuracy}

An accuracy of $0.35 \mathrm{~mm}$ RMS is quoted for the POLARIS on its data sheet. Our own measurements of the POLARIS accuracy for our tracked pointer (the tip of which is $180 \mathrm{~mm}$ from the origin of the POLARIS tool holder) are given in Table 1. It can be seen that the error in the $z$ coordinate, the depth-of-field coordinate, is greater than that in $x$ or $y$. As well, the tracking accuracy improves by a small amount when the tool holder is in the same orientation relative to the POLARIS for all measurements. 


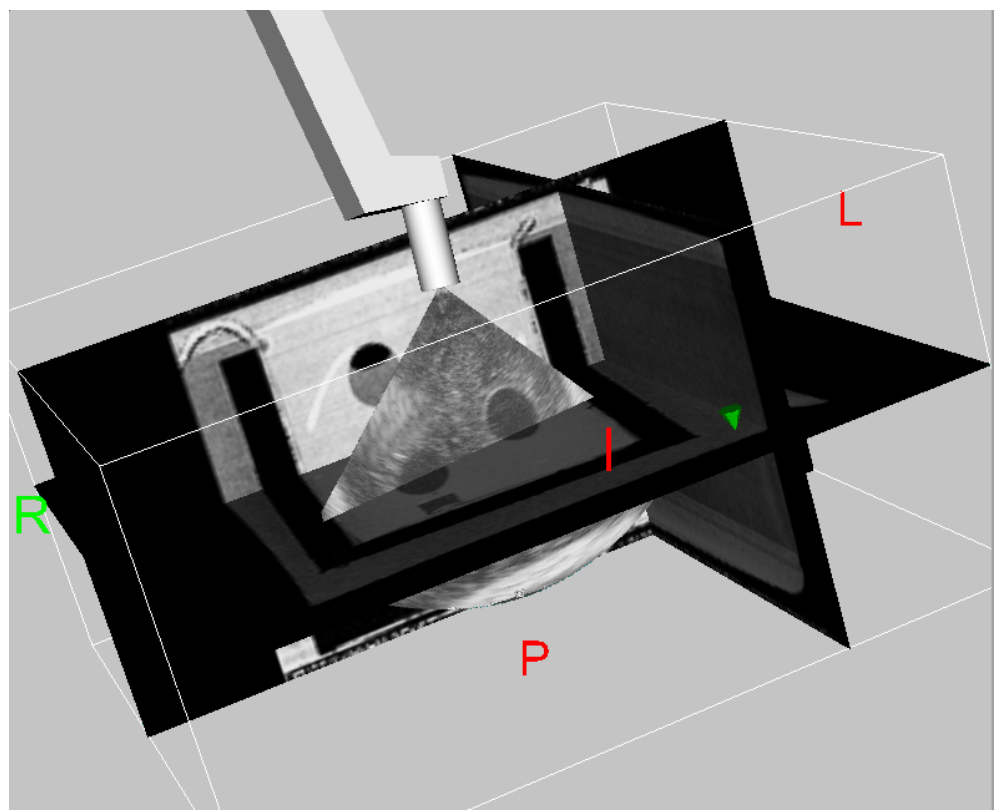

Fig. 3. The ultrasound video is texture-mapped into a $3 \mathrm{D}$ view of the MR data.

When several samples are averaged for each registration point, with a small change in the pointer orientation for each sample, the accuracy increases to $0.15 \mathrm{~mm}$. In comparison, fiducials in an MRI with $1 \mathrm{~mm}^{3}$ voxels can generally be located to within $0.3-1.0 \mathrm{~mm}$.

Table 1. Accuracy of the POLARIS Tracking System

\begin{tabular}{l|cccc}
\hline & $\begin{array}{c}\sigma_{x} \\
{[\mathrm{~mm}]}\end{array}$ & $\begin{array}{c}\sigma_{y} \\
{[\mathrm{~mm}]}\end{array}$ & $\begin{array}{c}\sigma_{x} \\
{[\mathrm{~mm}]}\end{array}$ & $\begin{array}{c}\sqrt{\sigma_{x}^{2}+\sigma_{y}^{2}+\sigma_{x}^{2}} \\
{[\mathrm{~mm}]}\end{array}$ \\
\hline \hline similar orientation & 0.093 & 0.114 & 0.282 & 0.318 \\
varied orientation & 0.157 & 0.190 & 0.316 & 0.401 \\
average 50 samples & 0.027 & 0.079 & 0.122 & 0.148 \\
\hline
\end{tabular}

This table is condensed from data included in [6]

\subsection{Ultrasound Calibration Accuracy}

The accuracy of the ultrasound probe calibration transform ${ }_{\mathrm{u}}^{\mathrm{h}} \mathrm{T}$ was estimated by performing four separate calibrations, using four ultrasound images for each calibration. For all four calibrations the calibration phantom was in the same 
location, and the phantom was only registered to the OTS once to determine the transformation ${ }_{\mathrm{f}}^{\mathrm{t}} T$. Because each of the fiducial divots was registered to within $0.15 \mathrm{~mm}$, the overall error on ${ }_{\mathrm{f}}^{\mathrm{t}} T$ was on the order of $0.2 \mathrm{~mm}$.

For each of the calibration matrices ${ }_{\mathrm{u}}^{\mathrm{h}} T_{i}$, a point $\mathbf{p}_{\mathrm{u}}$ in the ultrasound image was converted to tool holder coordinates $\mathbf{p}_{\mathrm{h} i}={ }_{\mathrm{u}}^{\mathrm{h}} T_{i} \mathbf{p}_{\mathrm{u}}$. The standard deviation $\sigma_{\mathrm{p}_{\mathrm{h}}}$ of these coordinates is the reproducibility of the calibration. This can be converted into ultrasound image coordinates through the transformation $\sigma_{\mathrm{p}_{\mathrm{u}}}={ }_{\mathrm{h}}^{\mathrm{u}} T \sigma_{\mathrm{p}_{\mathrm{h}}}$ where ${ }_{\mathrm{h}}^{\mathrm{u}} T$ is the inverse of any of the ${ }_{\mathrm{u}}^{\mathrm{h}} T$.



Fig. 4. Reproducibility of the ultrasound probe calibration.

Figure 4 shows the values of $\boldsymbol{\sigma}_{\mathbf{p}_{\mathrm{u}}}$ (broken into $x, y$, and $z$ components) for points along the $y$ axis (depth axis) of the ultrasound image. The values of $\sigma_{x}$ and $\sigma_{y}$ are excellent; $\sigma_{z}$ is comparable only at $3.5 \mathrm{~cm}$ depth (approximately 1 $\mathrm{cm}$ below the top $\mathrm{Z}$ bar in most of the images used for the calibration).

\subsection{Registration Accuracy}

The POLARIS tracking accuracy is $0.3-0.4 \mathrm{~mm}$, the accuracy of the registration of the calibration phantom to the OTS is $0.2 \mathrm{~mm}$, and the ultrasound probe calibration reproducibility is better than $0.3 \mathrm{~mm}$ in either $x$ or $y$. The total registration accuracy between ultrasound image coordinates and the OTS coordinates is $0.5 \mathrm{~mm}$ or better in $x, y$ and between 0.5 and 2.0 in $z$. The accuracy in an MR/ultrasound overlay is therefore governed primarily by the error in the registration of the MR, which is usually on the order of 1-2 mm. 


\section{Conclusion}

We have determined that the registration of ultrasound images for use in stereotactic neurosurgery can be done to a very high precision, better than $0.5 \mathrm{~mm}$ in the $x$ and $y$ directions and around $1 \mathrm{~mm}$ in the $z$ direction except at depths greater than $7 \mathrm{~cm}$. This precision is more than sufficient to accurately measure and correct for brain shift. In fact, the accuracy of the measurement of brain shift will be limited by either the accuracy of the registration of the MR images (via conventional stereotactic techniqes) or by the resolution of the $\mathrm{MR}$ and ultrasound images.

\section{Acknowledgments}

We gratefully acknowledge Kathleen Surry for building our PVA-cryogel phantom and Yves Starreveld for co-developing ASP. Funding for this research is provided by the Medical Research Council of Canada, and scholarship funding for David Gobbi is provided by the Ontario Ministry of Education.

\section{References}

1. D. L. G. Hill, C. R. Maurer Jr., R. J. Maciunas, J. A. Barwise, J. M. Fitzpatrick and M.Y. Wang. Measurement of Intraoperative Brain Surface Deformation under a Craniotomy. Neurosurgery 43:514-528, 1998.

2. D. W. Roberts, A. Hartov, F. E. Kennedy, M. I. Miga and K. D. Paulsen. Intraoperative Brain Shift and Deformation: A Quantitative Analysis of Cortical Displacement in 28 Cases. Neurosurgery 43:749-760, 1998.

3. R .D. Bucholz, D. Yeh,, J. Trobaugh, L. L. McDurmont, C. D. Sturm, C. Baumann and M. H. Jaimie. The Correction of Stereotactic Inaccuracy Caused by Brain Shift Using an Intraoperative Ultrasound Device. CVRMed-MRCAS '97' First Joint Conference Computer Vision, Virtual Reality and Robotics in Medicine and Medical Robotics and Computer-Assisted Surgery, Troccaz, J., Grimson, E., Mösges, R., (eds.), Grenoble, France, Springer-Verlag, Berlin, 1997.

4. C. Giorgi and D.S. Casolino. Preliminary Clinical Experience with Intraoperative Stereotactic Ultrasound Imaging. Stereotactic and Functional Neurosurgery 68:54$58,1997$.

5. A. Jödicke, W. Deinsberger, H. Erbe, A. Kriete and D.-K. Böker. Intraoperative Three-Dimensional Ultrasonography: An Approach to Register Brain Shift Using Multidimensional Image Processing. Minim. Invasive Neurosurg. 41:13-19, 1998.

6. R. M. Comeau, D. G. Gobbi, A. Fenster, A. F. Sadikot and T. M. Peters. Detecting and Correcting Brain Tissue Deformation Using Intraoperative Ultrasound Imaging in Interactive Image Guided Neurosurgery. Canadian Organization of Medical Physicists Conference Proceedings, 131-133, June 18-20, 1998.

7. R. M. Comeau, A. Fenster and T. M. Peters. Intraoperative US in Interactive ImageGuided Neurosurgery. Radiographics 18:1019-1027, 1998.

8. W. Schroeder, K. W. Martin and W. Lorensen. The Visualization Toolkit, 2nd Edition. Prentice Hall, Toronto, 1998.

9. I. Mano, H. Goshima, M. Nambu and I. Masahiro. New Polyvinyl Alcohol Gel Material for MRI Phantoms. Magnetic Resonance in Medicine 3: 921-926, 1986. 